\title{
Patologia do Ouvido Interno Imunomediada
}

\section{Immune-Mediated Inner Ear Disease}

Inês Gambôaํㄹ Delfim Duarte' ${ }^{1}$, Nuno Oliveira ${ }^{1}$

\section{RESUMO}

As doenças do ouvido interno de etiologia imune podem ocorrer como doença específica de órgão - doença autoimune do ouvido interno - ou no contexto de uma doença autoimune sistémica. O ouvido interno tem capacidade de resposta imune local, e como tal o dano vestibulococlear condicionado por estas patologias pode ocorrer por ativação de autoanticorpos, deposição de imunocomplexos ou fenómenos de vasculite. A etiologia da doença autoimune do ouvido interno não está ainda claramente definida, mas presume-se que a sua etiologia assente numa ativação de autoanticorpos dirigidos a proteínas do ouvido interno, sendo os mais estudados os anti-proteína de 68 kDa, anti-Hsp70 e anti-coclina. O desafio no diagnóstico deste tipo de patologia surge não só pela ausência de marcadores analíticos específicos, mas também pela grande variedade de patologias sistémicas que podem manifestar-se com sintomas audiovestibulares. A implementação de tratamento precoce é preponderante, uma vez que a perda auditiva pode ser reversível.

PALAVRAS-CHAVE: Doenças Autoimunes; Doenças do Labirinto; Fatores Imunológicos; Perda Auditiva Neurossensorial 


\begin{abstract}
Immune-mediated inner ear disease may occur as an organ-specific pathology - autoimmune inner ear disease - or in the context of a systemic autoimmune disease. This is because the inner ear can create a local immune response with vestibulocochlear damage that may occur due to autoantibody damage, immune complex deposition or vasculitis with microcirculation ischemia. The etiology of autoimmune inner ear disease is not yet clearly defined but the damage is presumed to be due to autoantibodies directed to inner ear proteins, the most studied being the anti-68 kDa, anti-Hsp70 and anti-cochlin proteins. The challenge in diagnosing this type of pathology arises not only because of the absence of specific analytical markers, but also because of the wide variety of systemic pathologies that may manifest with audiovestibular symptoms. However, early treatment is preponderant since hearing loss may be reversible.
\end{abstract}

KEYWORDS: Autoimmune Diseases; Hearing Loss, Sensorineural; Immunologic Factors; Labyrinth Diseases

\section{INTRODUÇÃO}

É reconhecido desde longa data que patologias imunomediadas podem afetar o ouvido interno ou o nervo vestibulococlear, com manifestações clínicas de surdez e vertigem. ${ }^{1}$ Alguns exemplos bem conhecidos de doenças sistémicas que podem atingir o ouvido interno incluem o lúpus eritematoso sistémico (LES), granulomatose de Wegener, síndrome de Cogan, vasculites primárias, entre outras. ${ }^{1,2}$ Algumas patologias do ouvido interno, tais como doença de Ménière, surdez súbita e labirintite poderão ter como base um mecanismo imunomediado, como sugerido na literatura atual. ${ }^{1-4}$

A doença do ouvido interno imunomediada surge mais frequentemente em adultos ou adultos jovens, apresentando-se como surdez neurossensorial bilateral simétrica ou assimétrica, progressiva (em semanas ou meses), flutuante ou súbita, e que melhora com a administração de corticoides ou outros imunossupressores. A perda auditiva pode ser acompanhada por vertigem ou acufeno. Os limiares auditivos podem também flutuar de acordo com a atividade da doença. ${ }^{1.5}$ Podem coexistir outros sintomas sistémicos ou de sistemas de órgãos em cerca de $30 \%$ dos casos. $2.5,6$

Esta orientação diagnóstica é importante, uma vez que podemos estar perante uma surdez neurossensorial potencialmente reversível e que pode ser a primeira manifestação de uma patologia sistémica. ${ }^{5}$

A patologia do ouvido interno de etiologia imune pode ser primária, ou órgão específica, de que é exemplo a doença autoimune do ouvido interno (DAOI), ou pode ser secundária, no contexto de outras doenças sistémicas imunomediadas. ${ }^{4,7}$

\section{MECANISMOS DE LESÃO IMUNOMEDIADA DO OUVIDO INTERNO}

O sistema imune atua contra patogénicos ambientais, distinguindo antigénios externos do hospedeiro. Eventos autoimunes ocorrem quando há um distúrbio no reconhecimento de antigénios do próprio organismo pelo sistema imune, sendo desencadeada uma resposta inflamatória com consequente lesão de tecidos e órgãos. ${ }^{1}$ Apesar de a etiologia precisa da DAOI não estar claramente definida, os mecanismos patológicos que podem explicar esta condição são semelhantes aos de outras doenças autoimunes - envolvimento de autoanticorpos com ativação de mediadores inflamatórios e resposta de células T CD4+. ${ }^{6}$ A impossibilidade de biópsia do ouvido interno para estudos histológicos dificulta o estudo dos mecanismos precisos de lesão nestas patologias, sendo os mecanismos hipotetizados baseados em estudos em animais, de autópsia ou estudos experimentais baseados na clínica e exames complementares de diagnóstico. ${ }^{2}$ Há, de facto, uma correlação significativa de estudos em animais com os achados serológicos em humanos. ${ }^{5}$

\section{COMO É, ENTÃO, GERADO O ESTIIMULO AUTOIMUNE NO OUVIDO INTERNO?}

Segundo Harris et al, o ouvido interno é capaz de gerar uma resposta imune local após ter ocorrido imunização a um antigénio local ou sistémico. ${ }^{8}$ Esta imunização pode ocorrer após eventos inflamatórios, traumáticos ou infeciosos comprometerem a barreira hematolabiríntica, possibilitando a infiltração de anticorpos e células $\mathrm{T}$ ativadas no ouvido interno.,4,9 $\mathrm{O}$ processamento de antigénios no ouvido interno ocorre no saco endolinfático, que possui células imunocompetentes. ${ }^{8}$ As células mediadoras têm acesso à rampa timpânica através da 
microcirculação, difundem-se pelo saco endolinfático e a reação inflamatória que geram vão levar a labirintite, com perda de células sensitivas e disfunção fisiológica, com progressão para fibrose e osteoneogénese coclear e vestibular. 1,3,4 $\mathrm{Na}$ DAOI a reação por autoanticorpos pode ser desencadeada quando o sistema imune atua contra danos externos. Vírus ou bactérias podem atingir diretamente o ouvido interno por via hematogénea, liquor ou através do ouvido médio, levando a alterações em moléculas do hospedeiro que se tornam autoantigénios, alvos de uma posterior resposta imune local. ${ }^{6} \mathrm{~A}$ geração de autoanticorpos do ouvido interno pode também ocorrer por reatividade cruzada com outros patogénios. ${ }^{5,10} \bigcirc$ ouvido interno pode ser também exposto a autoanticorpos após cirurgia ou traumatismos locais. ${ }^{6}$

Em doenças sistémicas o dano ao ouvido interno pode ocorrer por vários mecanismos. Pode haver deposição de imunocomplexos na microcirculação vestibulococlear com degeneração da estria vascular, sem necessidade de haver uma resposta inflamatória local - estudo em cobaias animais com LES. ${ }^{1}$ Podem ocorrer também fenómenos vasculíticos próprios da patologia de base, com isquemia e compromisso vascular, ${ }^{1,4}$ levando a fibrose e osteoneogénese coclear em fases finais da inflamação. 1,3 Sabe-se também que autoanticorpos dirigidos a epítopos cocleares podem ser patogénicos em doentes com uma suscetibilidade a autoimunidade. ${ }^{11}$ Existe, também, a possibilidade de lesão neuronal no nervo vestibulococlear. ${ }^{11}$

\section{DOENÇA AUTOIMUNE DO OUVIDO INTERNO (DAOI)}

Foi pela primeira vez descrita por Lehnhardt em 1958, que sugeriu a existência de anticorpos anti-cocleares como causa de surdez neurossensorial progressiva em 13 doentes. ${ }^{12}$ No entanto, a sua definição clássica foi feita por McCabe em 1979. ${ }^{13}$ Este autor reportou 18 casos de doentes com surdez neurossensorial rapidamente progressiva sem causa identificável, que obtiveram melhoria auditiva após tratamento com imunossupressores. A evidência de autoimunidade nestes doentes incluiu a positividade do teste de inibição de linfócitos. Neste teste, o soro dos pacientes foi combinado com antigénios do labirinto membranoso de doentes submetidos a ressecção translabiríntica de tumores acústicos, tendo havido reação com o soro de todos os pacientes, que foi negativa nos controlos.

A DAOI manifesta-se por surdez neurossensorial bilateral progressiva, em semanas ou meses, simétrica ou assimétrica, que responde à administração de corticoides e outros imunossupressores. ${ }^{1}$ Pode também apresentar-se como surdez neurossensorial súbita ou flutuante (semelhante a doença de Ménière). ${ }^{6}$ Pode ser inicialmente unilateral, podendo decorrer meses até a bilateralidade ocorrer. ${ }^{5}$ Cerca de $50 \%$ dos doentes podem ter sintomas vestibulares ${ }^{1,6,9}$ e cerca de $25 \%$ - 50\% podem ter acufenos e plenitude aural. ${ }^{5}$ É mais comum em mulheres, é rara em crianças, ${ }^{6}$ e pode estar associada a outras doenças sistémicas em cerca de 30\% dos casos. ${ }^{5}$ Representa menos de $1 \%$ de todas as causas de surdez neurossensorial e vestibulopatia. ${ }^{9}$ A sua verdadeira incidência é desconhecida devido à inexistência de testes diagnósticos específicos. ${ }^{9} \bigcirc$ diagnóstico assenta na avaliação clínica, com constatação de surdez neurossensorial progressiva no audiograma, havendo melhoria audiométrica após administração de corticoides ou outros imunossupressores. ${ }^{1}$ É importante neste contexto excluir outras causas de surdez neurossensorial com características clínicas semelhantes, como a sífilis, doença de Lyme, toxoplasmose, VIH ou exposição prévia a fármacos ototóxicos. ${ }^{1,5} \bigcirc$ diagnóstico pode ser suportado por testes serológicos com pesquisa de anticorpos anti-proteínas do ouvido interno (anti-68 kDa, anti-Hsp70 e anti-coclina), contudo, a sua sensibilidade de deteção é baixa, e a sua negatividade não exclui o diagnóstico. ${ }^{5}$

\section{ANTIGÉNIOS ALVO DA RESPOSTA IMUNE}

São hipotetizados vários antigénios alvo da resposta imune na DAOI.

Foi encontrado em humanos com DAOI um anticorpo específico que se liga a uma proteína de 68 kDa de osso temporal bovino. ${ }^{14}$ Este anticorpo também se liga à proteína de choque térmico de $70 \mathrm{kDa}-\mathrm{Hsp} 70 .{ }^{15} \mathrm{O}$ epítopo primário deste anticorpo anti-proteína de $68 \mathrm{kDa}$ não está ainda totalmente esclarecido, bem como o seu papel na DAOI. ${ }^{1}$

Alguns estudos que suportam a existência destes anticorpos incluem os trabalhos de Shin et al, ${ }^{16}$ onde é demonstrada a positividade para anticorpos anti-proteína de $68 \mathrm{kDa}$ em 40\% de doentes com surdez neurossensorial rapidamente progressiva, e de Boulassel et al, com $44 \%$ de doentes com DAOI com anticorpos anti-proteínas de 30, 42 e 68 kDa.

Há também noutros estudos evidência de positividade de anticorpos anti-proteína Hsp70 na DAOI. ${ }^{17}$ Mas a proteína Hsp70 não é específica do ouvido interno, e está também presente no rim e intestino. ${ }^{18}$ Foi também demonstrado que estes anticorpos podem estar presentes em doentes com surdez neurossensorial após exposição ao ruído. ${ }^{19}$ 
Um outro antigénio frequentemente sugerido como alvo da resposta imune na DAOI é a coclina. ${ }^{10} \mathrm{~A}$ coclina é um componente major da matriz extracelular do ouvido interno, expressa exclusivamente na cóclea e vestíbulo em humanos. ${ }^{9}$ No ouvido interno existem 3 isoformas glicosiladas com pesos de 40, 46 e 60 kDa e uma isoforma de 16 kDa (tomoproteína coclina na perilinfa). ${ }^{9}$ Apesar de ser a segunda proteína mais abundante no ouvido interno (depois do colagénio tipo II) a sua função é pouco compreendida - foi sugerido um papel na homeostasia estrutural do vestíbulo. ${ }^{9}$

Vários estudos na literatura têm suportado o papel da coclina na patologia autoimune do ouvido interno. Sabe-se que, em animais, a coclina está também presente no baço, nas células foliculares dendríticas, e que tem um importante papel na produção de anticorpos. Foi demonstrado que ratos knok-out para coclina tiveram maior suscetibilidade a infeções por Pseudomonas aeruginosa e Staphylococcus aureus, havendo assim sugestão de um possível papel da coclina na resposta imune a patogénios no ouvido interno.?

Baek et al ${ }^{19}$ detetaram anticorpos anti-coclina em doentes com DAOI comparativamente a controlos saudáveis e em doentes com trauma acústico ou presbiacusia.

No trabalho de Solares et al, ${ }^{18}$ ratos imunizados com anticorpos anti-coclina desenvolveram surdez neurossensorial após 6 semanas, tendo-se verificado infiltração linfocítica nos locais onde a colina é mais abundante ligamento espiral e rampa timpânica.

No trabalho de Pathak et al, ${ }^{10}$ foi demonstrada uma homologia da coclina com espécies de Aspegillus e Peniciliumm, e verificado que doentes com DAOI têm níveis elevados de anticorpos dirigidos a coclina e a fungos comparativamente a controlos saudáveis, sugerindo que a exposição a fungos pode desencadear uma resposta autoimune em doentes suscetíveis. Os autores hipotetizaram que certos fungos podem atuar como PAMP's e gerar uma resposta imune inata em doentes com suscetibilidade a DAOI.

Apesar de existirem vários trabalhos de investigação na literatura na busca de um antigénio específico, a verdade é que, na prática clínica, ainda não estão totalmente definidos quais os antigénios alvo envolvidos na DAOI. De facto, verifica-se que nem sempre estão presentes em doentes com DAOI, que podem ser positivos noutras patologias do ouvido interno, e que a sua negatividade não exclui o diagnóstico de DAOI. 6 Permanece, assim, a dúvida de se estes anticorpos serão uma causa ou uma consequência no processo patológico envolvido.

\section{ABORDAGEM DIAGNÓSTICA}

Um diagnóstico precoce e a implementação de tratamento atempado têm impacto no prognóstico auditivo do doente. $O$ diagnóstico de surdez neurossensorial de etiologia imune baseia-se fundamentalmente na avaliação clínica, com documentação audiométrica da perda auditiva e melhoria com administração de imunossupressores, como anteriormente referido. Um exame objetivo detalhado não só otorrinolaringológico, mas também com revisão de sintomas por sistemas de órgãos, é importante para despiste de patologia sistémica. ${ }^{2}$ Após exclusão de outras causas de diagnósticos diferenciais de surdez neurossensorial, deve ser realizado estudo analítico básico semelhante ao realizado na suspeita de doenças autoimunes ${ }^{1,2}$ : hemograma, função renal e hepática, estudo da coagulação, proteína C reativa, velocidade de sedimentação, anticorpos anti-nucleares, anti-fosfolipídeos, anti-DsDNA, anti SSa/SSb, fator reumatoide, doseamento de imunoglobulinas, doseamento do complemento e função tiroideia. Outros marcadores mais específicos podem ser pedidos de acordo com a suspeita clínica, sendo que a integração com outras especialidades médicas como Medicina Interna ou Reumatologia pode ser necessária. $\bigcirc$ doseamento de anticorpos anti-proteína de 68 KDa, anti-Hsp70 ou anti-coclina devem ser pedidos, de acordo com a disponibilidade, se bem que a sua negatividade não exclui o diagnóstico de DAOI. 5,6

Os estudos de imagem do ouvido interno podem demonstrar alterações que corroboram o diagnóstico. Na ressonância magnética, pode haver alterações inespecíficas no ouvido interno como estreitamento, calcificação ou obliteração dos tecidos moles no labirinto vestibular e cóclea. ${ }^{20}$ Por vezes há hipersinal em T1 no labirinto afetado como manifestação de inflamação aguda. ${ }^{21}$ Contudo, estas alterações não são específicas, e indicam apenas a existência de um processo inflamatório agudo ou crónico em estádios finais de inflamação que podem estar presentes em variadas patologias, como osteoneogénese e calcificação cocleovestibular. ${ }^{3,21}$ A tomografia de emissão de positrões (PET) tem sido usada recentemente para avaliar patologias infeciosas e inflamatórias com elevado metabolismo glicídico intra-celular. ${ }^{22}$ Num estudo, foram verificadas alterações de sinal no ouvido interno na PET em doentes com DAOI com positividade para anticorpos anti-HSP70 e em fases ativas da doença, com normalização do sinal após melhoria audiométrica. ${ }^{23}$ Apesar dos achados, a PET não deve ser usada como exame de rotina dado serem necessários mais estudos sobre a resolução do scan e o radioisótopo a ser usado. $^{23}$ 


\section{TERAPÊUTICA}

A introdução de terapêutica imunossupressora é fundamental para o prognóstico auditivo. A resposta positiva a corticoides sistémicos ocorre em cerca de 60\% dos doentes. ${ }^{6}$ Segundo as recomendações na literatura, deve ser iniciada com uma dose de $60 \mathrm{mg} /$ dia de prednisolona durante pelo menos 4 semanas. ${ }^{1,6}$ Uma resposta positiva é objetivada com audiograma, quando se observa uma diminuição dos limiares auditivos de $15 \mathrm{~dB}$ numa frequência, $10 \mathrm{~dB}$ em duas ou mais frequências ou meIhoria na discriminação. Doentes com flutuação auditiva podem ter apenas estabilização desta, sem melhoria dos limiares. ${ }^{1}$ Devem ser realizados audiogramas mensais, e a corticoterapia deve ser mantida em dose máxima até se verificar estabilização auditiva. $\bigcirc$ desmame éfeito em 8 semanas, até uma dose de manutenção de $10-20$ mg/ dia por tempo variável, sendo recomendável um tratamento com duração mínima de 6 meses. ${ }^{1,6}$ Em casos de resistência, dependência ou efeitos adversos da corticoterapia, podem ser usados outros imunossupressores como metotrexato, ciclofosfamida ou infliximab, descritos em vários casos na literatura. ${ }^{6}$

A terapêutica local intratimpânica pode ser uma alternativa ou complemento ao tratamento sistémico. Tem as vantagens de ser minimamente invasiva e de minimizar os efeitos adversos dos imunossupressores. Assim, quando indicado, pode ser feita com injeções semanais, durante 1 a 2 meses, de 0,3-0,5 mL de metilprednisolona a $40 \mathrm{mg} / \mathrm{mL}$ pela membrana timpânica intacta, sobre o nicho da janela redonda. ${ }^{6}$ Contudo, não existe ainda um consenso na literatura sobre a duração do tratamento nem os casos em que este tratamento é mais indicado. ${ }^{1}$

Nos casos de perda auditiva neurossensorial definitiva a reabilitação auditiva deve ser planeada. A utilização de implantes cocleares nestes casos pode ser uma alternativa. ${ }^{24}$ Contudo, devem ser considerados os riscos associados à terapêutica imunossupressora prolongada e à própria patologia de base, uma vez que há tendência para a doença inflamatória induzir uma reação endoestriatal com obliteração coclear por tecido cicatricial e osteoneogénese, que constitui um desafio para o implante coclear. Assim, é necessária uma avaliação detalhada para implantação antes da ocorrência de ossificação coclear para que os resultados auditivos sejam os melhores. ${ }^{24}$

\section{CONCLUSÃO}

A surdez neurossensorial imunomediada representa um desafio na prática clínica, uma vez que a ausência de tes- tes diagnósticos específicos implica que o diagnóstico assente num forte índice de suspeição. Apesar de serem reconhecidos alguns autoanticorpos que podem ter um papel na etiologia da DAOI, não há ainda um marcador analítico específico para esta patologia, e a negatividade para estes autoanticorpos não exclui o seu diagnóstico. O facto de o ouvido interno poder estar também envolvido numa grande variedade de patologias sistémicas imunomediadas torna importante a compreensão dos mecanismos de lesão imune e a suspeita clínica destas patologias pelo otorrinolaringologista. $\bigcirc$ tratamento deve ser implementado o mais precocemente possível, podendo ser necessária a integração com outras especialidades médicas na avaliação e seguimento do doente. Isto torna possível uma recuperação auditiva máxima ou, no caso de irreversibilidade, a prevenção de um agravamento ou a possibilidade de reabilitação.

CONFLITOS DE INTERESSE: Os autores declaram não ter qualquer conflito de interesse na realização do presente trabalho.

FONTES DE FINANCIAMENTO: Não houve qualquer fonte de financiamento na realização do presente trabalho.

CONFLICTS OF INTEREST: The authors declare that they have no conflicts of interest.

FINANCIAL SUPPORT: This work has not received any contribution, grant or scholarship.

\section{REFERÊNCIAS}

1. Brant JA, Ruckenstein MJ. Autoimmune Inner Ear Disease. In: Flint PW, Haughey BH, Lund VJ, Niparko JK, et al, editors. Cummings Otolaryngology \& Head and Neck Surgery. 6th ed. Philadelphia: Elsevier; 2015. p. 227-30.

2. Harris JP, Weisman MH. Head and Neck Manifestations of Systemic Disease. New York: Informa Healthcare USA; 2007.

3. Swartz JD, Hagiwara M. Inflammatory Diseases of the Temporal Bone. In: Som PM, Curtin HD, editors. Head and Neck Imaging. 5th ed. Missouri: Mosby; 2011. p.1183-1229.

4. Dorado JC, Fernández MP, Villarin SR, González-Gay MA. Audiovestibular manifestations in systemic vasculitis. Acta Otorrinolaringol Esp. 2009;60:432-42.

5. Bovo R, Ciorba A, Martini A. The diagnosis of autoimmune inner ear disease: evidence and clinical pitfalls. Eur Arch Otolaryngol. 2009;266:37-40. doi: 10.1007/s00405-008-0801-y.

6. Lobo DR, García-Berrocal JR, Ramirez-Camacho R. New prospects in the diagnosis and treatment of immune-mediated inner ear disease. World J Methodol. 2014;4:91-8. doi: 10.5662/wjm.v4.i2.91.

7. Buniel MC, Geelan-Hansen K, Weber PC, Tuohy VK. Immunosuppressive therapy for autoimmune inner ear disease. Immunotherapy. 2009;1:425-34. doi: 10.2217/imt.09.12

8. Harris JP, Heydt J, Keithley EM, Chen MC. Immunopathology of the inner ear: an update. Ann N Y Acad Sci. 1999;830:16678. 
9. Baruah P. Cochlin in autoimmune inner ear disease: Is the search for an inner ear autoantigen over? Auris Nasus Larynx. 2014;1:499-501. doi: 10.1016/j.anl.2014.08.014.

10. Pathak S, Hatam LJ, Bonagura V, Vambutas A. Innate immune recognition of molds and homology to the inner ear protein, cochlin, in patients with autoimmune inner ear disease. J Clin Immunol. 2013;33:1204-15. doi: 10.1007/s10875-0139926-x.

11. Tucci M, Quatraro C, Silvestris F. Sjogren's syndrome: an autoimmune disorder with otolaryngological involvement. Acta Otorhinolaryngol Ital. 2005;25:139-144.

12. Lehnhardt E. Sudden hearing disorders occurring simultaneously or successively on both sides. Z Laringol Rinol Otol. 1958;37:1-16.

13. McCabe BF. Autoimmune sensorineural hearing loss. Ann Otol Rhinol Laryngol. 1979;88:585-9.

14. Harris JP, Sharp PA. Inner ear autoantibodies in patients with rapidly progressive sensorineural hearing loss. Laryngoscope. 1990;100:516-24.

15. Billings PB, Keithley EM, Harris JP. Evidence linking the $68 \mathrm{ki}-$ lodalton antigen identified in progressive sensorineural hearing loss patient sera with heat shock protein 70. Ann Otol Rhinol Laryngol.1995;104:181-8.

16. Shin SO, Billings PB, Keithley EM, Harris JP. Comparison of anti-heat shock protein 70 (anti-hsp70) and anti-68-kDa inner ear protein in the sera of patients with Meniere's disease. Laryngoscope. 1997;107:222-7.

17. Boulassel MR, Deggouj N, Tomasi JP, Gersdorff M. Inner ear autoantibodies and their targets in patients with autoimmune inner ear diseases. Acta Otolaryngol. 2001;121:2834.

18. Solares CA, Edling AE, Johnson JM, Baek MJ, Hirose K, Hughes GB, et al. Murine autoimmune hearing loss mediated by CD4+ T cells specific for inner ear peptides. J Clin Invest. 2004;113:1210-7.

19. Baek MJ, Park HM, Johnson JM, Altuntas CZ, Jane-Wit D, Jaini $R$, et al. Increased frequencies of cochlin-specific $T$ cells in patients with autoimmune sensorineural hearing loss. J Immunol. 2006;177:4203-10.

20. Greco A, Gallo A, Fusconi M, Magliulo G, Turchetta R, Marinelli $C$, et al. Cogan's syndrome: an autoimmune inner ear disease. Autoimmun Rev. 2013;12:396-400. doi: 10.1016/j. autrev.2012.07.012.

21. Hegarty JL, Patel S, Fischbein N, Jackler RK, Lalwani AK. The value of enhanced magnetic resonance imaging in the evaluation of endocochlear disease. Laryngoscope. 2002;112:8 -17. doi: 10.1097/00005537-200201000-00002

22. Orsal E, Uğur M, Seven B, Ayan AK, Içyer F, Yıldız A. The importance of FDG-PET/CT in Cogan's syndrome. Mol Imaging Radionucl Ther. 2014;23:74-5. doi: 10.4274/mirt.349.

23. Mazlumzadeh M, Lowe VJ, Mullan BP, Fabry DA, McDonald TJ, Matteson EL. The utility of positron emission tomography in the evaluation of autoimmune hearing loss. Otol Neurotol. 2003;24:201-4.

24. Malik MU, Pandian V, Masood H, Diaz DA, Varela V, Dávalos-Balderas AJ, et al. Spectrum of immune-mediated inner ear disease and cochlear implant results. Laryngoscope. 2012;122:2557-62. oi: 10.1007/s12026-015-8696-3. 